\title{
Integrated Microwave Photonic Spectral Shaping for Linearization and Spurious-Free Dynamic Range Enhancement
}

\author{
Gaojian Liu, Okky Daulay, Yvan Klaver, Roel Botter, Qinggui Tan, Hongxi Yu, Marcel Hoekman, \\ Edwin J. Klein, and David Marpaung
}

(Invited Paper)

\begin{abstract}
Integrated microwave photonics (MWP) is a fast growing area where high frequency microwave signals are processed in the optical domain, merging key advantages of both microwave photonics and photonic integrated circuits (PICs) technologies including low-loss, reconfigurability, advanced functionality, enhanced stability, and reduced footprint. Plenty of functionalities have been demonstrated in integrated MWP, especially based on spectral shaping technique, where the phase and amplitude of the optical spectrum is precisely tailored by PICs. However, on-chip linearization is lagging behind and has not been investigated deeply. It is crucial and urgent to study on-chip linearization methods, which will lead to advanced integrated MWP systems with large spurious-free dynamic range (SFDR). In this paper, we present two novel techniques for on-chip linearization of microwave photonic links. The first technique is based on line-by-line complex spectral shaping using a series of ring resonators. The second technique relies on spatial separation to achieve parallel spectral shaping in two complementary spatial channels. Both methods are demonstrated in low-loss programmable silicon nitride circuits that can already host a number of advanced functionalities. Our results point to the great potential of integrating advanced functionalities and linearization in the same integrated platform.
\end{abstract}

Index Terms - Integrated microwave photonics, optical spectral shaping, linearization, spurious-free dynamic range.

\section{INTRODUCTION}

$\mathrm{M}$ ICROWAVE photonics (MWP) is a field that merges the microwave and photonics technologies and has great potential for the high frequency, broad bandwidth application in 5G communication, satellite communication, radio astronomy and radar system [1]-[3]. The use of photonic devices to process and distribute the radio frequency (RF) signal in MWP systems, brings key advantages such as large bandwidth, reconfigurability, low loss, and electromagnetic interference immunity.

Originally, MWP systems are based on the discrete fiber-

Date of submission: April 14, 2021.

This work was supported by Netherlands Organisation for Scientific Research NWO Vidi (15702) and Start Up (740.018.021). Gaojian Liu was supported by Chinese Scholarship Council. (Corresponding author: David Marpaung)

G. Liu is with Nonlinear Nanophotonics - LPNO Group, University of Twente, Enschede, The Netherlands, and also with China Academy of Space Technology (Xi'an), Xi'an 710100, China (e-mail: g.liu@utwente.nl). optic devices, which are bulky and sensitive to external perturbation [4]. Nowadays, with the convergence of photonic integrated circuits (PICs) and MWP, integrated MWP becomes the key technology which can dramatically reduce the footprint of the MWP system, enable complex advanced functionalities, and improve the stability of the system [4], [5]. This makes MWP a real instrumental solution for electronic bottlenecks in microwave engineering.

Plenty of advanced functionalities have been achieved in integrated MWP, such as tunable filters [6]-[13], delay lines and beamforming [14]-[17], RF waveform generation [18][21], and instantaneous frequency measurement [22], [23]. Moreover, several studies have been done to improve the figure of merit of integrated MWP system, such as improving the link gain[11], and reducing the noise figure [12], [13]. However, on-chip linearization has not been investigated deeply.

Spurious free dynamic range (SFDR) is an important figure of merit in MWP system, used to describe the linearity of the system. Its upper limit is determined by the intermodulation terms in the system, especially third-order intermodulation (IMD3). Because the IMD3 is too close to the fundamental signal and cannot be filtered out by a filter. This requires techniques to cancel the IMD3 components through interferometric linearization techniques.

So far, linearization techniques have not been looked at too much on-chip. An early work of on-chip SFDR enhancement was proposed and demonstrated by Marpaung et al. with a chip based frequency discriminator [24]. The frequency discriminator consists of 5 tunable ring resonators with two mirrored output response, yielding a linear phase modulation (PM) to intensity modulation (IM) conversion, which leads to an improved SFDR. Most other linearization techniques have been done in fiber-based systems, either by creating an auxiliary path to generate complementary IMD3 terms which will be subtracted at the photodetector [25]-[29] or by directly

O. Daulay, Y. Klaver, R. Botter and D. Marpaung are with Nonlinear Nanophotonics - LPNO Group, University of Twente, Enschede, The Netherlands (e-mail: o.f.p.daulay@utwente.nl; y.klaver@utwente.nl; r.a.botter@utwente.nl; david.marpaung@utwente.nl).

Q. Tan and H. Yu are with China Academy of Space Technology (Xi'an), Xi'an 710100, China (e-mail: uestctqg@163.com; yuhongxi123@aliyun.com).

M. Hoekman, E. J. Klein are with the LioniX International BV, Enschede AL 7500, The Netherlands (e-mail: m.hoekman@lionix-int.com; e.j.klein@lionixint.com). 
manipulating the phase and amplitude of the modulated optical spectrum to reduce the IMD3 components [30]-[35], namely spectral shaping. The auxiliary path can be established based on the different modulation efficiency of modulators in different wavelengths [25], different modulation efficiency in orthogonal polarization states [26], [27], and different modulation indices with different RF input power [28], [29]. These methods need either a pair of lasers or a pair of modulators, which increases the complexity of the system.

The spectral shaping method tailors the phase and amplitude of the modulated optical spectrum to make the IMD3 terms generated by beating between different optical sidebands interfere destructively at PD. This method is first implemented in intensity-modulation (IM) links. If a proper phase shift is imposed at the optical carrier band (OCB), which contains the optical carrier and the nonlinear distortion terms at each side of the carrier, the IMD3 terms from different contributors in the optical spectrum can cancel each other out [30], [33], [35].

Recently, the PM systems have gained significant interests in application, as the phase modulator is free from bias drifting problem and has a simpler structure with lower insertion loss, compared with intensity modulator. In PM links, a processing of phase modulation to intensity modulation (PM-IM) conversion is needed for direct detection. This processing generates the IMD3 in the system. With optical spectral shaping for IMD3 suppression, the phase-modulated direct-detection (PM-DD) links can be linearized and show a better linearity performance compared with the typical IM links. The linearization of a PM-DD link can be achieved by tailoring the power ratio between OCB and different optical sidebands (OSBs) in a single sideband phase modulated link [31], [36] or tuning the phase of OCB and \pm 2 OSB simultaneously [32]. If multi-order sidebands are taken into account and both phase and amplitude are manipulated, IMD3 terms from third-order, fifthorder and even higher order nonlinearity can be suppressed simultaneously, leading to a further improved SFDR [34]. Among these spectral shaping methods, the optical spectra are manipulated by liquid crystal on silicon (LCoS) processer [30], [32], [34], external optical filter and attenuator [36], stimulated Brillouin scattering effect [33] or dual-parallel Mach-Zehnder modulator (DPMZM) [35]. The systems are still bulk and complex.

It is important to achieve linearization with a PIC, especially in the scheme that share the same building blocks with the advanced functionalities, so that the linearization and advanced functionalities can be achieved in the same integrated platform, which will greatly reduce the footprint and the complexity of linearized system [37].

In this paper, we present our recent works about on-chip linearization methods. Both methods are based on the novel technique of integrated microwave photonic spectral shaping that we recently introduced which use PICs to vectorially manipulate the modulated optical spectra [37], [38]. The first technique is an in-line scheme that uses a series of cascaded ring resonators for line-by-line shaping of the phase and amplitude of optical spectrum to reduce the IMD3 terms [39]. The second approach is based on spatial separation of the RF modulated optical spectra in a new silicon nitride MWP spectral shaping circuit architecture [38]. The optical spectra are processed parallelly to improve the linearity. In the proof of concept experiments the IMD3 suppression of $22.4 \mathrm{~dB}$ and $20.3 \mathrm{~dB}$ are observed for the respective techniques. These works open the avenue for on-chip linearization in MWP system and shows the potential of integrating advanced functionalities and linearization in the same PIC.

\section{SERIAL SPECTRAL SHAPING FOR LINEARIZATION}

A serial spectral shaping scheme for linearization is shown in Fig. 1. A programmable $\mathrm{Si}_{3} \mathrm{~N}_{4}$ chip with four all-pass ring resonator is used to shape the optical spectrum line-by-line to suppress the IMD3 terms. The phase and amplitude shaping imposed to optical spectrum is determined by the amplitude response $T(\omega)$ and phase response $\theta(\omega)$ of ring resonators, which can be tuned by thermo-optic effect. The response can be expressed as

$$
T(\omega) e^{j \theta(\omega)}=\frac{a-c e^{-j \phi(\omega)}}{1-a c e^{j \phi(\omega)}} e^{j[\pi+\phi(\omega)]}
$$

In (1), $c=\sqrt{1-k}, a=10^{-\alpha L / 20}, k, \alpha$ and $\phi$ are selfcoupling coefficient, single-pass amplitude of the optical ring resonator, power coupling coefficient, propagation loss of optical waveguide $(\mathrm{dB} / \mathrm{cm})$, and round-trip phase respectively. The all-pass ring resonator can be configured in three coupling states. When $\mathrm{c}=\mathrm{a}$, it operates at critical-coupling (CC) state. When $c>a$ or $c<a$, it works at under-coupling (UC) or overcoupling (OC) state respectively. The UC ring exhibits a phase shift of 0 degree at the resonant frequency, while the OC ring produces a 180-degrees phase shift at resonance and has a successive phase transition from 0 to 360 degrees around the resonance.

As illustrated in Fig. 1, a two-tone RF signal is modulated to the optical carrier via a PM. The modulated optical spectrum consists of OCB and several OSBs. The modulated optical signal is then coupled into the programmable $\mathrm{Si}_{3} \mathrm{~N}_{4}$ chip for optical spectrum processing after amplification. First, only ring 1 at $\mathrm{OC}$ state is used to impose 90 degrees phase shift at OCB for PMIM conversion. After processing, the RF fundamental signal can be directly detected at photodetector (PD) with relatively large IMD3 terms. This state of the link is measured as reference state without linearization. Then, all the four rings on the chip are used for PM-IM conversion and linearization. Ring 1 at OC state and ring 4 at UC state are used to process OCB. Ring 2 and ring 3 at OC state are used to process \pm 2 OSB. After carefully adjusting the amplitude suppression and phase shift at OCB and \pm 2 OSB, the IMD 3 components generated from beating between different optical bands can interfere destructively at photodetector (PD).

The relation between optical spectrum and components of photo-current detected by $\mathrm{PD}$ needs to be investigated to determine the amplitude suppression and phase shift imposed to 


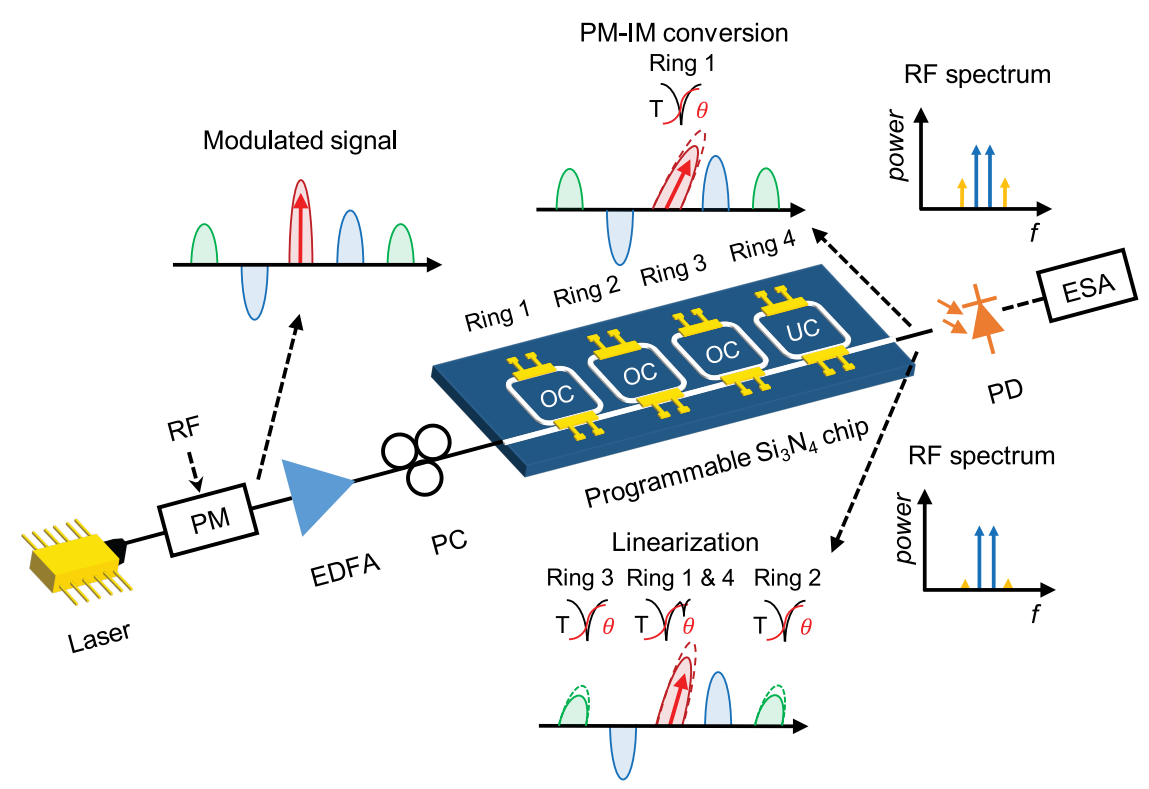

Fig. 1. Schematic of the proposed MWP link. PM, phase modulator; PC, polarization control; OC, over-coupling; UC, under-coupling; EDFA, erbium-doped fiber amplifier; PD, photodetector; ESA, electronic spectrum analyzer [39].

optical spectrum for linearization. The output optical spectrum of PM with a two-tone test can be expressed as

$$
E_{\text {out }}(t)=\sqrt{P_{i}} e^{j \omega_{c} t} \sum_{n=-\infty}^{+\infty} J_{n}(m) e^{j n \omega_{1} t} \sum_{k=-\infty}^{+\infty} J_{k}(m) e^{j k \omega_{2} t}
$$

where $\omega_{\mathrm{c}}, \omega_{1}, \omega_{2}, P_{i}, J_{n}, m=\pi V_{R F} / V_{\pi, R F}, V_{R F}$, and $V_{\pi, R F}$ is the angular frequency of the optical carrier, the input two-tone RF signal, input optical power, the $n$-th order Bessel function of the first kind, the modulation index of PM, the voltage of input RF signal, and the RF half-wave voltage of the PM respectively. Under small signal condition, only zero to second order sidebands are taken into account for approximation.

The output optical spectrum of PM is depicted in Fig. 2, which consists of the OCB, the \pm 1 OSB and the \pm 2 OSB. The OCB is composed of the optical carrier and the even-order nonlinear terms at each side of the carrier. The \pm 1 OSB contains the fundamental signal and odd-order distortion terms. The \pm 2 OSB contains the even-order nonlinear components. There are three pairs of the main contributors for the IMD3. The

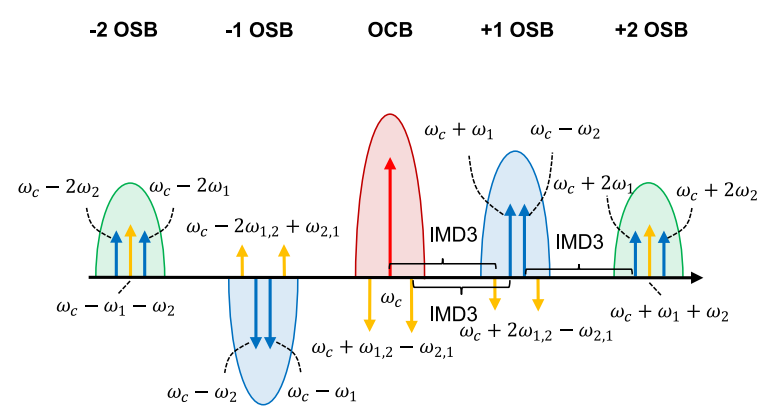

Fig. 2. Output optical spectrum of PM under two-tone test [39]. first one is the beating of the optical carrier $\omega_{c}$ and $\omega_{c}+$ $2 \omega_{1,2}-\omega_{2,1}\left(\omega_{c}-2 \omega_{1,2}+\omega_{2,1}\right)$. The second one is the beating between \pm 1 OSB of $\omega_{c}+\omega_{1,2}\left(\omega_{c}-\omega_{1,2}\right)$ and \pm 2 OSB of $\omega_{c}+2 \omega_{2,1}\left(\omega_{c}-2 \omega_{2,1}\right)$. The beating product between $\omega_{c}+\omega_{2,1}-\omega_{1,2}\left(\omega_{c}-\omega_{2,1}+\omega_{1,2}\right)$ and $\omega_{c}+\omega_{1,2}$ $\left(\omega_{c}-\omega_{1,2}\right)$ is the third contributor. The sum of three beating products is the dominant part of the IMD3 components in a PM link.

Optical field after processing by programmable $\mathrm{Si}_{3} \mathrm{~N}_{4}$ chip with four ring resonators can be written as

$$
E_{p}(t)=\sqrt{P_{i}} e^{j \omega_{c} t}\left\{\begin{array}{l}
T_{1}(\omega) T_{4}(\omega) e^{j\left[\theta_{1}(\omega)+\theta_{4}(\omega)\right]} J_{0} J_{0} \\
+T_{1}(\omega) T_{4}(\omega) e^{j\left[\theta_{1}(\omega)+\theta_{4}(\omega)\right]} \\
J_{-1} J_{1}\left[e^{-j\left(\omega_{1}-\omega_{2}\right) t}+e^{j\left(\omega_{1}-\omega_{2}\right) t}\right] \\
+J_{0} J_{1}\left(e^{j \omega_{1} t}+e^{j \omega_{2} t}\right) \\
+J_{-1} J_{2}\left[e^{j\left(2 \omega_{1}-\omega_{2}\right) t}+e^{j\left(2 \omega_{2}-\omega_{1}\right) t}\right] \\
+J_{-1} J_{0}\left(e^{-j \omega_{1} t}+e^{-j \omega_{2} t}\right) \\
+J_{-2} J_{1}\left[e^{-j\left(2 \omega_{1}-\omega_{2}\right) t}+e^{-j\left(2 \omega_{2}-\omega_{1}\right) t}\right] \\
+T_{2}(\omega) e^{j \theta_{2}(\omega)} J_{0} J_{2}\left(e^{j 2 \omega_{1} t}+e^{j 2 \omega_{2} t}\right) \\
+T_{2}(\omega) e^{j \theta_{2}(\omega)} J_{1} J_{1} e^{j\left(\omega_{1}+\omega_{2}\right) t} \\
+T_{3}(\omega) e^{j \theta_{3}(\omega)} J_{-2} J_{0}\left(e^{-j 2 \omega_{1} t}+e^{-j 2 \omega_{2} t}\right) \\
+T_{3}(\omega) e^{j \theta_{3}(\omega)} J_{-1} J_{-1} e^{-j\left(\omega_{1}+\omega_{2}\right) t}
\end{array}\right\}
$$

where $J_{n}=J_{n}(m)(\mathrm{n}=0, \pm 1, \pm 2), J_{-n}=(-1)^{n} J_{n}$. Amplitude 
response $T_{i}(\omega)$ and phase response $\theta_{i}(\omega)$ of the $i$-th ring resonator are given in (1).

In our spectrum shaping process the amplitude suppression and phase shift imposed to the \pm 2 OSB are the same, which means $T_{2}=T_{3}$ and $\theta_{2}=\theta_{3}$. Then the processed optical signal is sent to PD to retrieve RF signal. The photo-current at the output of PD can be written as

$$
\begin{aligned}
I_{P D}(t) & =R_{P D}\left|E_{P}(t)\right|^{2} \\
& =I_{1} \cos \left(\omega_{1,2} t\right)+I_{3} \cos \left(2 \omega_{1,2} t-\omega_{2,1} t\right)
\end{aligned}
$$

where $R_{P D}$ is the responsivity of photodetector, $I_{1}$ and $I_{3}$ are the coefficients for fundamental signal and IMD3 components, which can be expressed as

$$
\begin{gathered}
I_{1}=4 R_{P D} P_{i} T_{1} T_{4} J_{0}^{3} J_{1} \sin \left(\theta_{1}+\theta_{4}\right) \\
I_{3}=-4 R_{P D} P_{i}\left[\begin{array}{l}
T_{1} T_{4} J_{0}^{2} J_{1} J_{2} \sin \left(\theta_{1}+\theta_{4}\right) \\
+T_{2} J_{0}^{2} J_{1} J_{2} \sin \theta_{2} \\
+T_{1} T_{4} J_{0} J_{1}^{3} \sin \left(\theta_{1}+\theta_{4}\right)
\end{array}\right]
\end{gathered}
$$

After applying small-signal approximation for the Bessel function, $J_{n}(m) \approx m^{n} /\left(2^{n} n\right.$ !) for $m \ll 1, I_{l}$, and $I_{3}$ can be described as

$$
\begin{gathered}
I_{1} \approx 2 R_{P D} P_{i} m T_{1} T_{4} \sin \left(\theta_{1}+\theta_{4}\right) \\
I_{3} \approx-R_{P D} P_{i} \frac{m^{3}}{4}\left[3 T_{1} T_{4} \sin \left(\theta_{1}+\theta_{4}\right)+T_{2} \sin \theta_{2}\right]
\end{gathered}
$$

The IMD3 distortion caused by third-order nonlinearity in system will be minimized when the processing imposed to the OCB and \pm 2 OSB satisfies the condition:

$$
3 T_{1} T_{4} \sin \left(\theta_{1}+\theta_{4}\right)+T_{2} \sin \theta_{2}=0
$$

To minimize the IMD3 and maximize the fundamental signal simultaneously, in our spectral shaping process, we carefully tune the ring response to make $\sin \left(\theta_{1}+\theta_{4}\right)=-1 / 3, \sin \theta_{2}=$ $1, T_{1} T_{4}=T_{2}$, which means $\arcsin (1 / 3) \approx 340.5^{\circ}$ and $90^{\circ}$ phase shifts are imposed to OCB and \pm 2 OSB respectively, and the amplitude suppression at OCB and \pm 2 OSB are equal. When the condition in (9) is met, $I_{3}$ is greatly suppressed. However, in the meantime, $I_{l}$ will be also reduced to $1 / 3$ of the original value according to (7). This leads to a $9.54 \mathrm{~dB}$ power penalty of fundamental signal.

An experiment is performed to verify our theoretical analysis. The setup is illustrated in Fig.1. A continuous-wave (CW) light from a laser (Pure Photonics PPCL550) operating at $1550 \mathrm{~nm}$ with an output power of $18 \mathrm{dBm}$ is modulated by a phase modulator (Thorlabs $10 \mathrm{GHz}$ ) with a two-tone RF signal centered at $5.005 \mathrm{GHz}$ with a $10 \mathrm{MHz}$ frequency separation. The modulator has an optical insertion loss of $4 \mathrm{~dB}$. The twotone signal with power of $3 \mathrm{dBm}$ is generated by signal generators (Wiltron 69147A and Rohde-Schwarz SMP02). The output of PM is amplified by a $2 \mathrm{~W}$ erbium-doped fiber amplifier (EDFA, Amonics), and then sent to a programmable silicon nitride chip (LioniX International)[40] for optical spectrum processing. The fiber-to-fiber insertion loss of the chip is $8.5 \mathrm{~dB}$ and the propagation loss of the waveguide is 0.15 $\mathrm{dB} / \mathrm{cm}$. The chip contains four cascaded all-pass ring resonators with a free spectral range (FSR) of $25 \mathrm{GHz}$ and Q factor of $6.09 \times 10^{5}$ at critical coupling state. The coupling coefficient and resonance frequency of ring resonators can be tuned by using heaters to change the effective index of the waveguide. The processed optical spectrum is then sent to a photodetector (APIC $40 \mathrm{GHz}$ Photodetector) to retrieve RF signal. The RF signal after photodetection is sent to an RF spectrum analyzer (Keysight N9000B) for measurement.

The measurement results of RF spectrum are shown in Fig.3. The fundamental to IMD3 ratio without linearization is $32.6 \mathrm{~dB}$ as depicted in Fig. 3(a). After linearization, a fundamental to IMD3 ratio of $55.0 \mathrm{~dB}$ is achieved which is $22.4 \mathrm{~dB}$ better than the reference link, as shown in Fig. 3(b). The power of fundamental signal is decreased by $10.2 \mathrm{~dB}$ after linearization, which fits the analysis. The power penalty can be compensated by increasing the output power of laser or EDFA. The SFDR of the proposed MWP link is measured in Fig.4. The measured

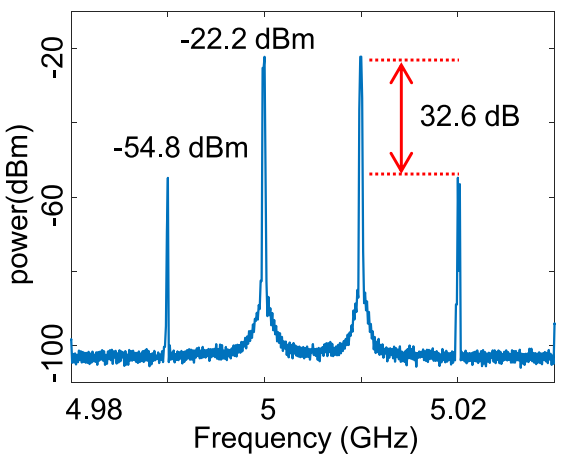

(a)

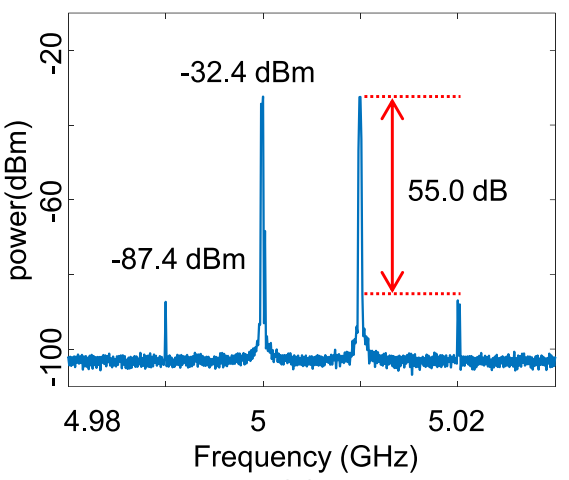

(b)

Fig. 3. Experiment result of proposed linearization method (a) RF spectrum without linearization (b) RF spectrum with linearization [39]. 


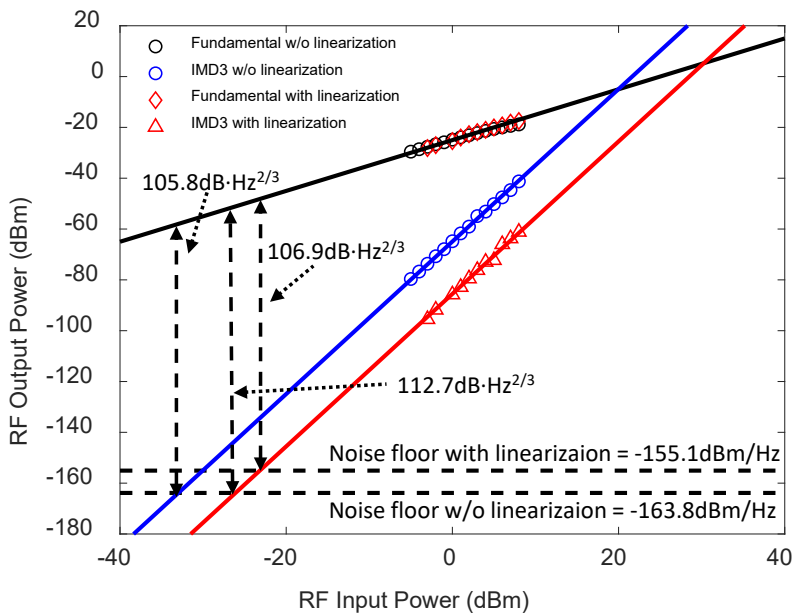

Fig. 4. SFDR of the MWP link before linearization and after linearization with gain compensation [39].

fundamental RF power and IMD3 power in $\mathrm{dBm}$ are fitted linearly with a fixed slope of 1 and 3 respectively to extract the SFDRs and input third order intercept point (IIP3). The SFDR of the link before linearization is $105.8 \mathrm{~dB} \cdot \mathrm{Hz}^{2 / 3}$ with a measured noise floor of $-163.8 \mathrm{dBm} / \mathrm{Hz}$, and the linearized link is $106.9 \mathrm{~dB} \cdot \mathrm{Hz}^{2 / 3}$ with a measured noise floor of -155.1 $\mathrm{dBm} / \mathrm{Hz}$. The increase of noise floor is mainly due to the increased optical power impinging to the photodetector. If the power penalty of fundamental signal can be compensated without elevating the noise floor, the SFDR of the linearized link can reach $112.7 \mathrm{~dB} \cdot \mathrm{Hz}^{2 / 3}$, which is $6.9 \mathrm{~dB}$ improvement.

To compare the linearity performance between the proposed PM link and the IM link, we extract the IIP3 from the measured data and calculate the IIP3 of an intensity modulator with the same $V_{\pi, R F}$ as $5 \mathrm{~V}$ using the formula $I I P 3_{I M}=4 V_{\pi, R F}^{2} /\left(\pi^{2} R\right)$, where $R=50 \Omega$ [41]. The IIP3 of the proposed PM link is improved from $20.0 \mathrm{dBm}$ to $30.3 \mathrm{dBm}$ after linearization. The IIP3 of IM link is $23.1 \mathrm{dBm}$. A better linearity performance is achieved in the proposed linearized PM link.

\section{Parallel Spectral Shaping For Linearization}

A linearization method based on parallel spectral shaping is proposed in this section. The schematic of the method is illustrated in Fig. 5 (a). A reconfigurable $\mathrm{Si}_{3} \mathrm{~N}_{4}$ chip with a deinterleaver cascaded by an attenuator at each output port is used to manipulate the optical spectra. The image of the chip is shown in Fig. 5 (b). There are also four all-pass ring resonators and an output de-interleaver cascaded on the chip which are not activated in the experiment. The de-interleaver is based on a 3 rings loaded asymmetric Mach-Zehnder interferometer structure [42], which exhibits a box-shaped, flat-top complementary filter response at two output ports. The variable attenuator is implemented by a balanced MZI with a phase shifter at one arm. The complex transfer function of deinterleaver can be written as

$$
\left[\begin{array}{c}
H_{\text {bar }}(z) \\
H_{\text {cross }}(z)
\end{array}\right]=H_{T C 2}\left[\begin{array}{cc}
H_{r 1}(z) H_{r 3}(z) & 0 \\
0 & H_{r 2}(z) H_{\Delta L}(z)
\end{array}\right] H_{T C 1}
$$

where

$$
\begin{gathered}
H_{T C i}=\frac{1}{2} \cdot\left[\begin{array}{cc}
e^{-j \varphi_{i}}-1 & -j\left(e^{-j \varphi_{i}}+1\right) \\
-j\left(e^{-j \varphi_{i}}+1\right) & -e^{-j \varphi_{i}}+1
\end{array}\right] \\
H_{r i}\left(z_{r i}\right)=\frac{c_{r i}-a_{r i} e^{-j \varphi_{r i}} z_{r}^{-1}}{1-c_{r i} e^{-j \varphi_{r i}} a_{r i} z_{r}^{-1}} \\
H_{\Delta L}(z)=a_{\Delta L} e^{-j \varphi_{\Delta L}} z_{\Delta L}^{-1}
\end{gathered}
$$

The $\varphi_{i}, \varphi_{r i}, \varphi_{\Delta L}$ are the additional phase shift on one arm of the tunable coupler, ring resonator and one arm of the MZI, $c_{r i}=\sqrt{1-k_{r i}}, a=10^{-\alpha L / 20}, k_{r i}$, and $\alpha$ are self-coupling coefficient, single-pass amplitude attenuation of the optical ring resonator or waveguide, power coupling coefficient of ring, and propagation loss of optical waveguide $(\mathrm{dB} / \mathrm{cm})$ respectively. $z^{-1}=\exp \left(-j \omega n_{g} L / c\right), L_{r}=2 L_{\Delta L}$, where $n_{g}$ is the group index, $c$ is the speed of light in vacuum.

The transfer function of de-interleaver can be decomposed into amplitude responses $T_{b a r}(\omega), T_{\text {cross }}(\omega)$ and phase responses $\theta_{\text {bar }}(\omega), \theta_{\text {cross }}(\omega)$ for simplicity in the following analysis.

$$
\left[\begin{array}{c}
T_{\text {bar }}(\omega) e^{j \theta_{\text {bar }}(\omega)} \\
T_{\text {cross }}(\omega) e^{j \theta_{\text {crass }}(\omega)}
\end{array}\right]=\left[\begin{array}{c}
H_{\text {bar }}(z) \\
H_{\text {cross }}(z)
\end{array}\right]
$$

The phase modulated signal is first sent to an on-chip deinterleaver to separate the lower sideband and upper sideband with optical carrier. Then, the upper sideband with optical carrier are attenuated to make the IMD3 term generated from the beating products in upper sideband the same amplitude as the IMD3 terms from the lower sideband. The attenuated upper sideband with the optical carrier and the lower sideband are recombined at a balanced photodetector (BPD) to cancel the IMD3 terms.

The phase modulated two-tone signal is expressed in (2). Ideally, a well-designed de-interleaver has high isolation between two complementary channels. In this way, if we align the lower sideband of PM spectrum at passband of bar port response and upper sideband with carrier at passband of cross port response, we can assume that only lower sideband spectrum or upper sideband with carrier exists at the 


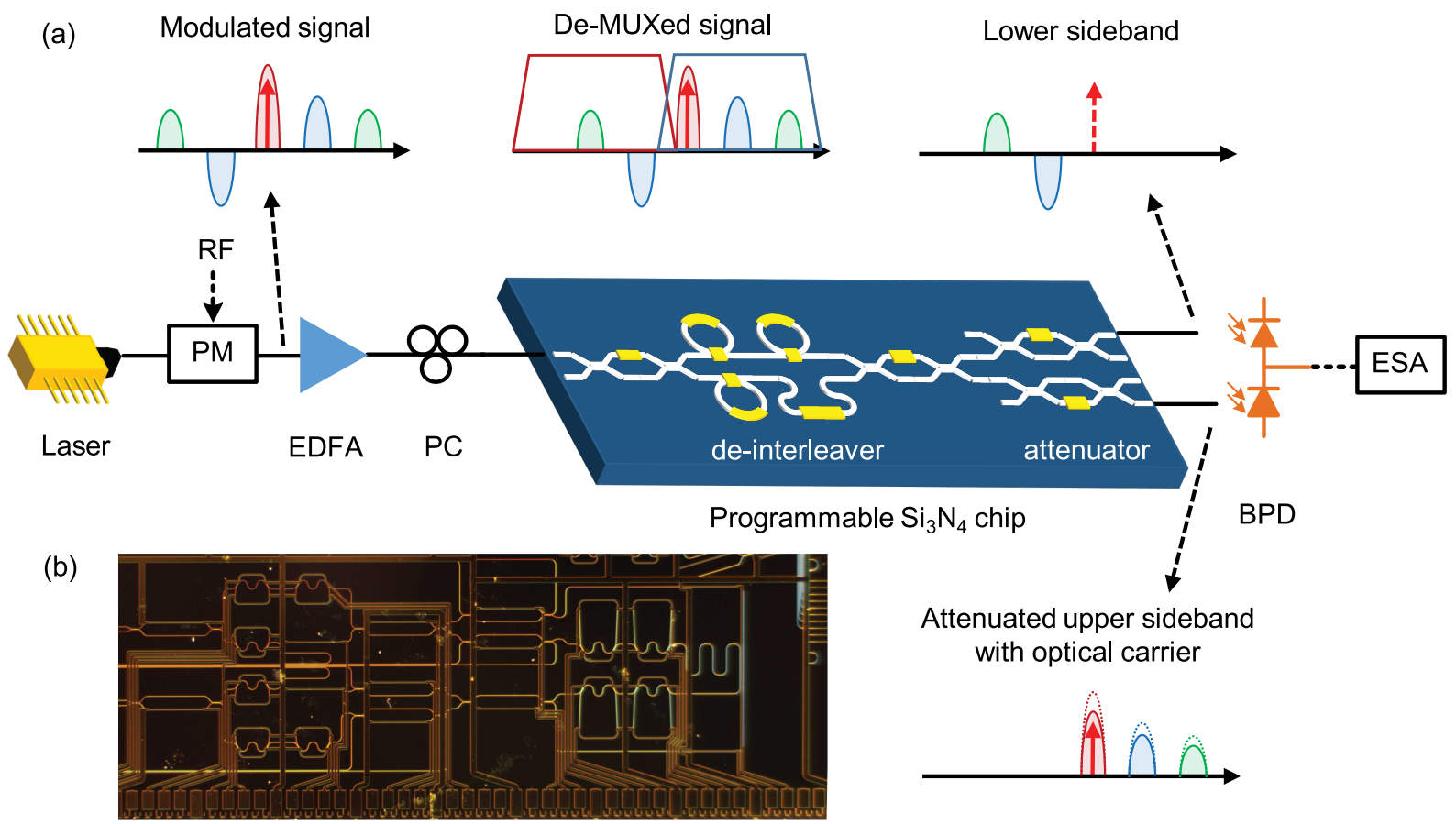

Fig. 5. (a) Schematic of spatial separated linearization method. PM, phase modulator; PC, polarization control; EDFA, erbium-doped fiber amplifier; BPD, balanced photodetector; ESA, electronic spectrum analyzer. (b) Image of the $\mathrm{Si}_{3} \mathrm{~N}_{4}$ chip (only the de-interleaver and attenuator at the top left of image are activated in the experiment).

corresponding output port. After the on-chip processing, the lower sideband optical spectrum and upper sideband with optical carrier can be expressed as (15) and (16) respectively.

$$
E_{L S B}(t)=\sqrt{P_{i}} e^{j \omega_{c} t}\left\{\begin{array}{l}
T_{b a r}\left(\omega_{c}-\omega_{1,2}\right) e^{j \theta_{b a r}\left(\omega_{c}-\omega_{1,2}\right)} \\
\cdot J_{-1} J_{0}\left(e^{-j \omega_{1} t}+e^{-j \omega_{2} t}\right) \\
+T_{b a r}\left(\omega_{c}-2 \omega_{1,2}+\omega_{2,1}\right) e^{j \theta_{b a r}\left(\omega_{c}-2 \omega_{1,2}+\omega_{2,1}\right)} \\
\cdot J_{-2} J_{1}\left[e^{-j\left(2 \omega_{1}-\omega_{2}\right) t}+e^{-j\left(2 \omega_{2}-\omega_{1}\right) t}\right] \\
+T_{b a r}\left(\omega_{c}-2 \omega_{1,2}\right) e^{j \theta_{b a r}\left(\omega_{c}-2 \omega_{1,2}\right)} \\
\cdot J_{-2} J_{0}\left(e^{-j 2 \omega_{1} t}+e^{-j 2 \omega_{2} t}\right) \\
+T_{b a r}\left(\omega_{c}-\omega_{1}-\omega_{2}\right) e^{j \theta_{b a r}\left(\omega_{c}-\omega_{1}-\omega_{2}\right)} \\
\cdot J_{-1} J_{-1} e^{-j\left(\omega_{1}+\omega_{2}\right) t}
\end{array}\right\}
$$

$$
E_{U S B}(t)=\sqrt{\beta P_{i}} e^{j \omega_{c} t}\left\{\begin{array}{l}
T_{\text {cross }}\left(\omega_{c}\right) e^{j \theta_{\text {cross }}\left(\omega_{c}\right)} J_{0} J_{0} \\
+T_{\text {cross }}\left(\omega_{c}-\omega_{1,2}+\omega_{2,1}\right) \\
e^{j \theta_{\text {cross }}\left(\omega_{c}-\omega_{1,2}+\omega_{2,1}\right)} \\
\cdot J_{-1} J_{1}\left[e^{-j\left(\omega_{1}-\omega_{2}\right) t}+e^{j\left(\omega_{1}-\omega_{2}\right) t}\right] \\
+T_{\text {cross }}\left(\omega_{c}+\omega_{1,2}\right) e^{j \theta_{\text {crosss }}\left(\omega_{c}+\omega_{1,2}\right)} \\
J_{0} J_{1}\left(e^{j \omega_{1} t}+e^{j \omega_{2} t}\right) \\
+T_{\text {cross }}\left(\omega_{c}+2 \omega_{1}-\omega_{2}\right) e^{j \theta_{\text {cross }}\left(\omega_{c}+2 \omega_{1}-\omega_{2}\right)} \\
J_{-1} J_{2}\left[e^{j\left(2 \omega_{1}-\omega_{2}\right) t}+e^{j\left(2 \omega_{2}-\omega_{1}\right) t}\right] \\
+T_{\text {cross }}\left(\omega_{c}+2 \omega_{1,2}\right) e^{j \theta_{\text {cross }}\left(\omega_{c}+2 \omega_{1,2}\right)} \\
J_{0} J_{2}\left(e^{j 2 \omega_{1} t}+e^{j 2 \omega_{2} t}\right) \\
+T_{\text {cross }}\left(\omega_{c}+\omega_{1}+\omega_{2}\right) e^{j \theta_{\text {cross }}\left(\omega_{c}+\omega_{1}+\omega_{2}\right)} \\
J_{1} J_{1} e^{j\left(\omega_{1}+\omega_{2}\right) t}
\end{array}\right\}
$$

where $\beta$ is the power attenuation in upper sideband.

In principle, the complementary channels have flat-top and identical amplitude response in the passband, so an assumption of $T_{\mathrm{bar}}(\omega)=T_{\text {cross }}(\omega)=T_{\text {pass }}$ can be made, where $T_{\text {pass }}$ describes the insertion loss in the passband of de-interleaver. Then the photocurrent at each photodiode of balanced detector can be written as: 


$$
I_{L S B}=2 R_{\text {pd }} P_{i} T_{\text {pass }}^{2}\left[\begin{array}{l}
-J_{0}^{2} J_{1} J_{2} \cos \left(\omega_{1,2} t+\Delta \theta_{\text {bar }} 1\right) \\
-J_{0} J_{1}^{3} \cos \left(\omega_{1,2} t+\Delta \theta_{\text {bar__ }}\right) \\
-J_{0}^{2} J_{1} J_{2} \cos \left(\left(2 \omega_{1,2}-\omega_{2,1}\right) t+\Delta \varphi_{\text {bar_ } 1}\right)
\end{array}\right]
$$

where

$$
\begin{aligned}
& \Delta \theta_{b a r_{-} 1}=\theta_{b a r}\left(\omega_{c}-\omega_{1,2}\right)-\theta_{b a r}\left(\omega_{c}-2 \omega_{1,2}\right) \\
& \Delta \theta_{b a r_{-} 2}=\theta_{b a r}\left(\omega_{c}-\omega_{2,1}\right)-\theta_{b a r}\left(\omega_{c}-\omega_{1}-\omega_{2}\right) \\
& \Delta \varphi_{b a r_{-} 1}=\theta_{b a r}\left(\omega_{c}-\omega_{2,1}\right)-\theta_{b a r}\left(\omega_{c}-2 \omega_{1,2}\right)
\end{aligned}
$$

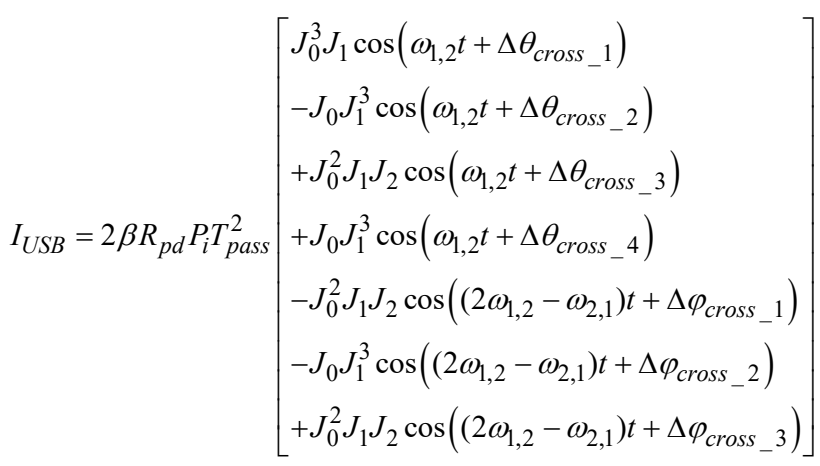

where

$$
\begin{aligned}
& \Delta \theta_{\text {cross_1 }}=\theta_{\text {cross }}\left(\omega_{c}+\omega_{1,2}\right)-\theta_{\text {cross }}\left(\omega_{c}\right) \\
& \Delta \theta_{\text {cross_2 }}=\theta_{\text {cross }}\left(\omega_{c}+\omega_{2,1}\right)-\theta_{\text {cross }}\left(\omega_{c}+\omega_{2,1}-\omega_{1,2}\right) \\
& \Delta \theta_{\text {cross_3 }}=\theta_{\text {cross }}\left(\omega_{c}+2 \omega_{1,2}\right)-\theta_{\text {cross }}\left(\omega_{c}+\omega_{1,2}\right) \\
& \Delta \theta_{\text {cross_4 }}=\theta_{\text {cross }}\left(\omega_{c}+\omega_{1,2}+\omega_{2,1}\right)-\theta_{\text {cross }}\left(\omega_{c}+\omega_{2,1}\right) \\
& \Delta \varphi_{\text {cross_1 }}=\theta_{\text {cross }}\left(\omega_{c}+2 \omega_{1,2}-\omega_{2,1}\right)-\theta_{\text {cross }}\left(\omega_{c}\right) \\
& \Delta \varphi_{\text {cross_2 }}=\theta_{\text {cross }}\left(\omega_{c}+\omega_{1,2}\right)-\theta_{\text {cross }}\left(\omega_{c}+\omega_{2,1}-\omega_{1,2}\right) \\
& \Delta \varphi_{\text {cross_3 }}=\theta_{\text {cross }}\left(\omega_{c}+2 \omega_{1,2}\right)-\theta_{\text {cross }}\left(\omega_{c}+\omega_{2,1}\right)
\end{aligned}
$$

In general, the de-interleaver has identical phase responses in the passband of complementary channels, and the phase response can be regarded as linear response at the center of passband. Then, we have $\Delta \theta_{\text {bar_}_{-} 1}=\Delta \theta_{\text {bar } 2_{-}}=\Delta \varphi_{\text {bar_1 }}=$ $\Delta \theta_{\text {bar }} \quad, \quad \Delta \theta_{\text {cross_1 }}=\Delta \theta_{\text {cross_2 }}=\Delta \theta_{\text {cross_3 }}=\Delta \theta_{\text {cross_4 }}=$ $\Delta \varphi_{\text {cross_ } 1}=\Delta \varphi_{\text {cross_ } 2}=\Delta \varphi_{\text {cross_ } 3}=\Delta \theta_{\text {cross }}$, and $\Delta \theta_{\text {bar }}=$ $\Delta \theta_{\text {cross }}=\Delta \theta$.

Then (17), (18) can be rewritten as

$$
\begin{gathered}
I_{L S B}=2 R_{p d} P_{i} T_{\text {pass }}^{2}\left[\begin{array}{l}
\left(-J_{0}^{2} J_{1} J_{2}-J_{0} J_{1}^{3}\right) \cos \left(\omega_{1,2} t+\Delta \theta\right) \\
-J_{0}^{2} J_{1} J_{2} \cos \left(\left(2 \omega_{1,2}-\omega_{2,1}\right) t+\Delta \theta\right)
\end{array}\right] \\
I_{U S B}=2 \beta R_{\text {pd }} P_{i} T_{\text {pass }}^{2}\left[\begin{array}{l}
\left(J_{0}^{3} J_{1}+J_{0}^{2} J_{1} J_{2}\right) \cos \left(\omega_{1,2} t+\Delta \theta\right) \\
-J_{0} J_{1}^{3} \cos \left(\left(2 \omega_{1,2}-\omega_{2,1}\right) t+\Delta \theta\right)
\end{array}\right]
\end{gathered}
$$

The photocurrent at output of BPD is

$$
\begin{aligned}
I_{\text {out }}=I_{U S B}-I_{L S B} \\
=2 R_{\text {pd }} P_{i} T_{\text {pass }}^{2}\left[\begin{array}{l}
\left(\beta J_{0}^{3} J_{1}+(\beta+1) J_{0}^{2} J_{1} J_{2}+J_{0} J_{1}^{3}\right) \\
\cdot \cos \left(\omega_{1,2} t+\Delta \theta\right) \\
+\left(-\beta J_{0} J_{1}^{3}+J_{0}^{2} J_{1} J_{2}\right) \\
\cdot \cos \left(\left(2 \omega_{1,2}-\omega_{2,1}\right) t+\Delta \theta\right)
\end{array}\right]
\end{aligned}
$$

Considering small signal approximation, when $m \ll 1$, $J_{n}(m) \approx m^{n} /\left(2^{n} n !\right), I_{\text {out }}$ can be written as

$$
I_{\text {out }}=R_{\text {pd }} P_{i} T_{\text {pass }}^{2}\left[\begin{array}{l}
\left(\beta m+\frac{3+\beta}{8} m^{3}\right) \cos \left(\omega_{1,2} t+\Delta \theta\right) \\
+\left(-\frac{\beta}{4}+\frac{1}{8}\right) m^{3} \cos \left(\left(2 \omega_{1,2}-\omega_{2,1}\right) t+\Delta \theta\right)
\end{array}\right]
$$

The IMD3 terms can be cancelled in the condition of $\beta=$ $1 / 2$, which means the optical power of upper sideband signal with carrier should be attenuated for $3 \mathrm{~dB}$.

An experiment is performed to demonstrate the effectiveness of the proposed method in Fig. 5 (a). A CW laser (Pure Photonics PPCL550) is set at $1549.945 \mathrm{~nm}$ with an output power of $16 \mathrm{dBm}$. Then, a two-tone RF signal with frequency of $14 \mathrm{GHz}$ and $14.01 \mathrm{GHz}$ and power of $3.5 \mathrm{dBm}$ from signal generators (Wiltron 69147A and Rohde-Schwarz SMP02) is modulated onto the optical carrier via a PM (Thorlabs $40 \mathrm{GHz}$ ). After amplified by a low noise erbium-doped fiber amplifier (EDFA, Amonics), the optical signal is sent to a programmable silicon nitride chip (LioniX International) for spectral shaping. The coupling loss of the chip was measured to be $1.5 \mathrm{~dB}$ per facet, the propagation loss of the straight waveguide is 0.1 $\mathrm{dB} / \mathrm{cm}$ and the effective propagation loss in ring resonator is $0.42 \mathrm{~dB} / \mathrm{cm}$. The increased effective propagation loss in the ring is due to the small bending radius $(80 \mu \mathrm{m})$ and loss at coupling section. The $\mathrm{Q}$ factor of ring resonator in de-interleaver at critical coupling state is $3.6 \times 10^{5}$. The FSR of the rings in deinterleaver is $80 \mathrm{GHz}$, and the de-interleaver has an FSR of 160 GHz. The processed optical signal is then sent to a BPD (DSC710), The retrieved RF signal after photodetection is sent to a RF spectrum analyzer (Keysight N9000B) for analysis.

First, only the upper sideband with optical carrier filtered out in cross port of de-interleaver is sent to one port of the BPD (this is regarded as main port of the BPD), this single sideband phase modulated signal is measured as the reference for the IMD3 suppression and SFDR improvement. Then an attenuator cascaded at cross port of de-interleaver is used to attenuate the amplitude of upper sideband with carrier. After carefully tuning the attenuation to meet the condition of $\beta=1 / 2$, the upper sideband with carrier in cross port and the lower sideband in bar port are recombined via a balanced detector. As the IMD3 terms from beating products in lower sideband and upper sideband with carrier are the same amplitude and in phase, they can be 


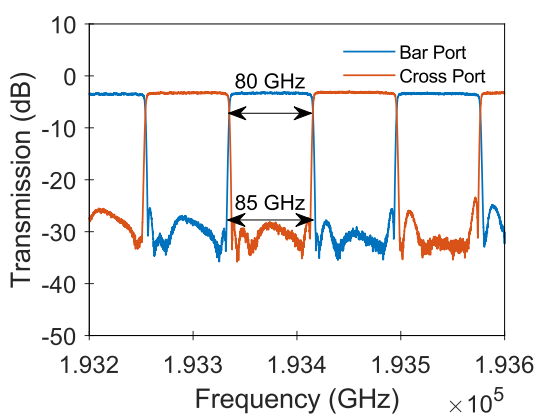

(a)

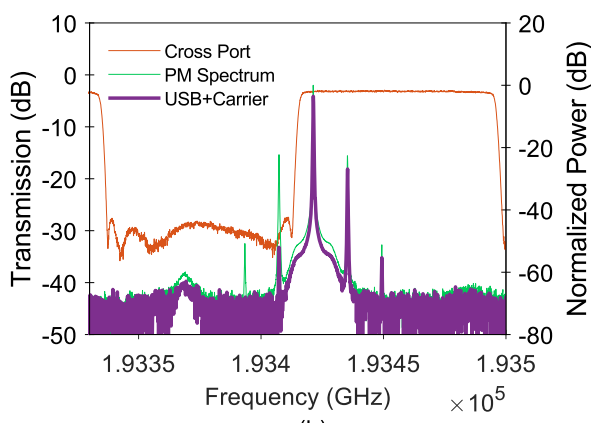

(b)

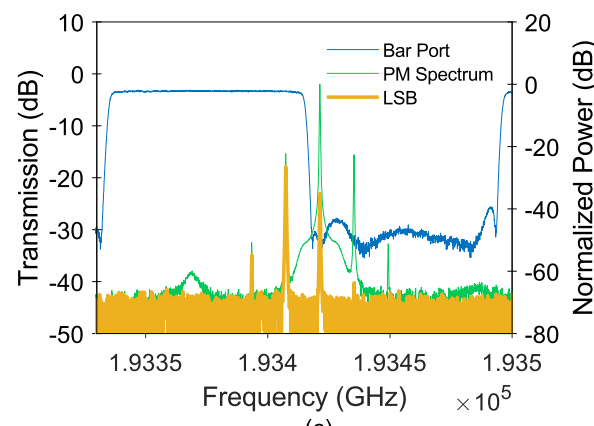

(c)

Fig. 6. De-interleaver response and the optical spectra at each output port of de-interleaver. (a) measured de-interleaver response (b) upper sideband (USB) with carrier (c) lower sideband (LSB). The modulated optical signal is normalized to the optical carrier power of the phase modulated signal at the input of de-interleaver.

cancelled out at the output of the BPD. The fundamental signals at the output of the BPD is mainly dominated by the beating products from upper sideband, and has a power penalty coefficient of $\beta^{2}=1 / 4$. To compensate the power penalty at fundamental signal, the output power of EDFA is increased to keep the optical power sent to main port of BPD the same as the reference link. In our experiment, the optical power sent to the main port of BPD maintains $10 \mathrm{dBm}$ before and after linearization.

The response of the on-chip de-interleaver and the separated optical spectrum are shown in Fig. 6. The de-interleaver has a 3 $\mathrm{dB}$ bandwidth of $80 \mathrm{GHz}$, and a suppression of $25 \mathrm{~dB}$ in stopband with a steep edge. The $3 \mathrm{~dB}$-to- $25 \mathrm{~dB}$ transition is measured as $2.5 \mathrm{GHz}$ which is equivalent to $1.6 \%$ of the FSR. The separated upper sideband with carrier and lower sideband spectra are shown in Fig. 6 (b) and (c) with optical spectrum at in put of de-intrerleaver as reference.

The RF spectrum retrieved from the upper sideband with the carrier is shown in Fig. 7 (a). The fundamental-to-IMD3 ratio (FIR) was measured to be $42 \mathrm{~dB}$. This state is the reference before linearization. The lower sideband spectra which mainly contains the first and second order sideband is sent to the complimentary PD in the BPD. The resulting RF spectrum after photodetection is shown in Fig. 7 (b). The IMD3 terms from this part will be used to cancel the IMD3 from the upper sideband. Then both the optical signal from upper and lower

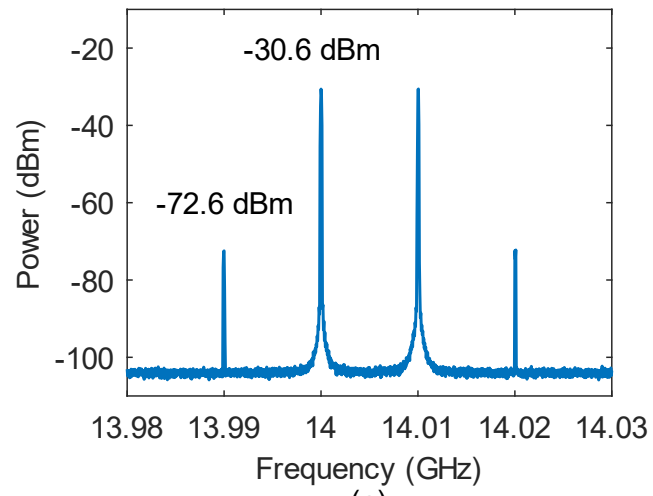

(a)

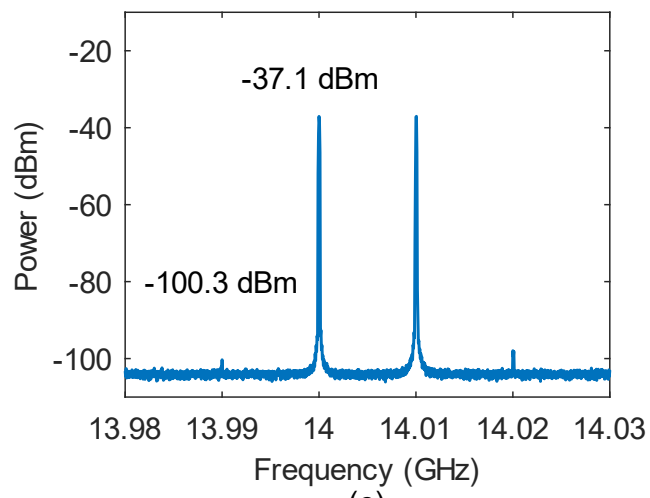

(c)

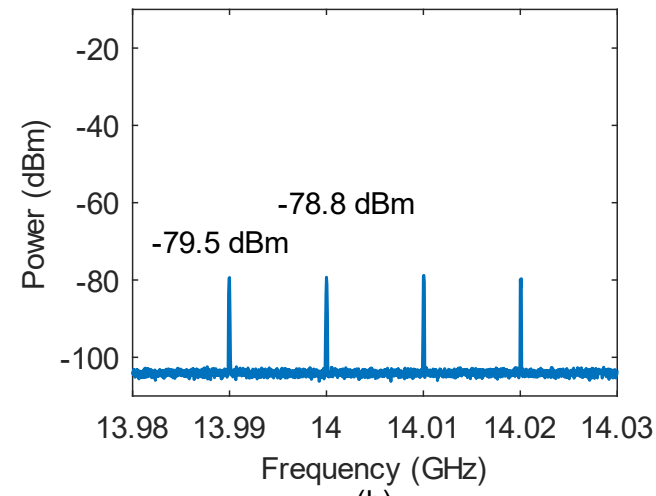

(b)

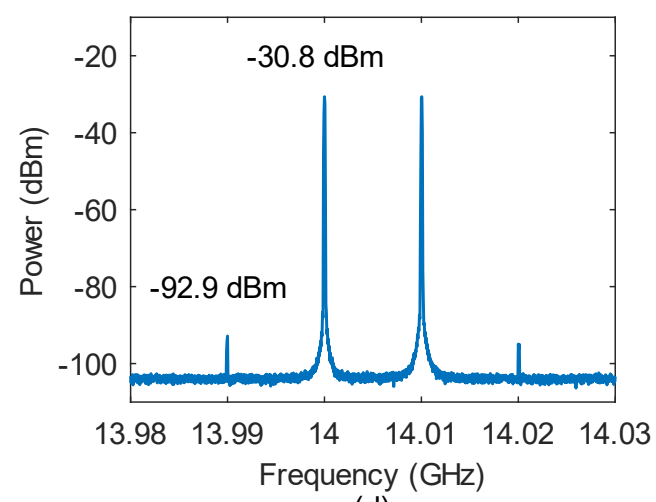

(d)

Fig. 7. Measured electrical spectrum (a) from upper sideband with carrier (b) from lower sideband (c) with linearization (d) with linearization and gain compensation. 


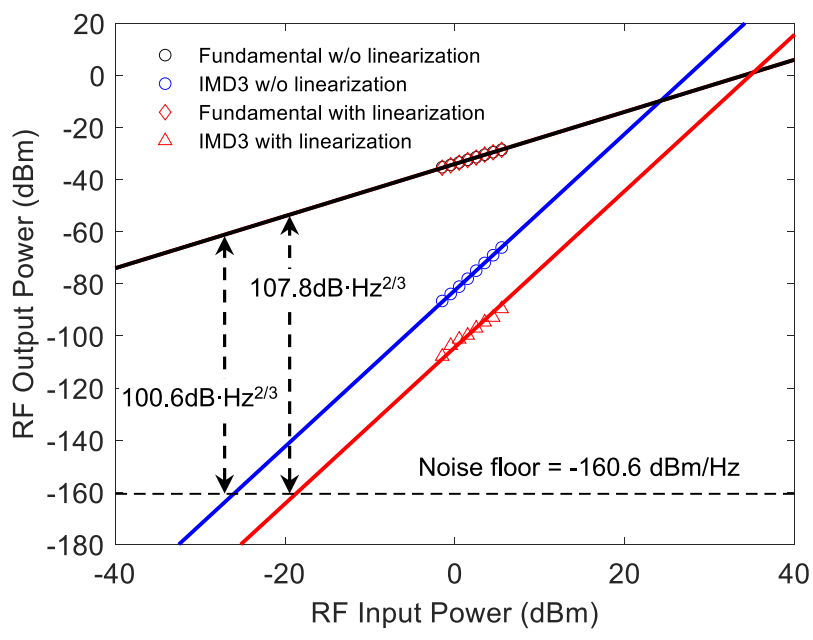

Fig. 8. Measured SFDR of MPL before and after linearization.

sideband are sent to the BPD. After attenuating the upper sideband spectrum for $3.5 \mathrm{~dB}$ in optical power, the IMD3 is suppressed clearly, and FIR is improved to $63.2 \mathrm{~dB}$, as shown in Fig. 7 (c).

The optical carrier and the upper sideband signal are attenuated to meet the condition of IMD3 cancellation, so there is a power penalty at fundamental signal of $6.5 \mathrm{~dB}$ in the measurement. This penalty can be compensated by increasing the output power of EDFA to keep the optical power sent to the BPD the same as reference state before linearization, as depicted in Fig. 7 (d). After the compensation, the FIR maintains $62.1 \mathrm{~dB}$, and a IMD3 suppression of $20.3 \mathrm{~dB}$ is observed.

SFDR of the proposed linearized link is measured and compared with the link where only the upper sideband with carrier is sent to the PD. As depicted in Fig 8, the SFDR of the link without linearization is $100.6 \mathrm{~dB} \cdot \mathrm{Hz}^{2 / 3}$, with a noise floor of $-160.6 \mathrm{dBm} / \mathrm{Hz}$. After linearization, the SFDR is improved to $107.8 \mathrm{~dB} \cdot \mathrm{Hz}^{2 / 3}$, while the noise floor maintains the same. An improvement of $7.2 \mathrm{~dB}$ is observed. The IIP3 without and with linearization can be estimated as $24.2 \mathrm{dBm}$ and $35.2 \mathrm{dBm}$. Compared with the calculated IIP3 of $26.0 \mathrm{dBm}$ in the IM link with the same $V_{\pi, R F}$ as $7 \mathrm{~V}$, the proposed linearized PM link shows a better performance in linearity.

\section{DISCUSSION}

\section{A. Limits of IMD3 suppression}

The IMD3 suppression in serial and parallel spectral shaping linearization methods are $22.4 \mathrm{~dB}$ and $20.3 \mathrm{~dB}$ respectively, however, the IMD3 power is increasing with a slope of 3 instead of 5, when the input RF power is increased. This means the IMD3 caused by third order nonlinearity is not eliminated completely.

In the serial spectrum shaping scheme, degradation in IMD3 suppression is mainly because the amplitude suppression and phase shift imposed by ring resonators are not constant in processing bandwidth and are not independent as in the case of LCoS signal processor. These lead to limitations in perfectly

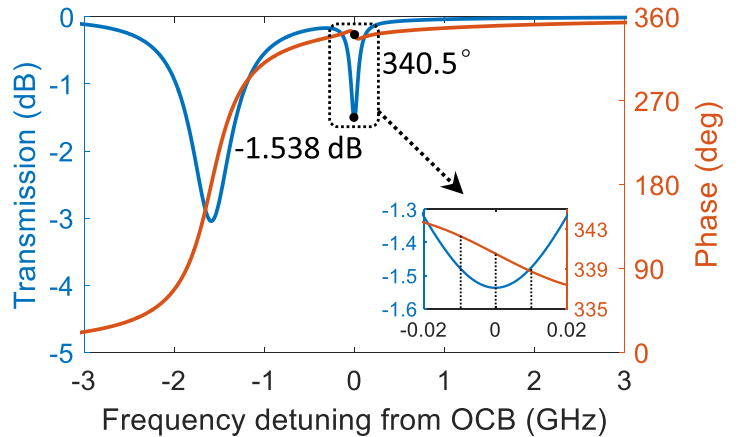

(a)

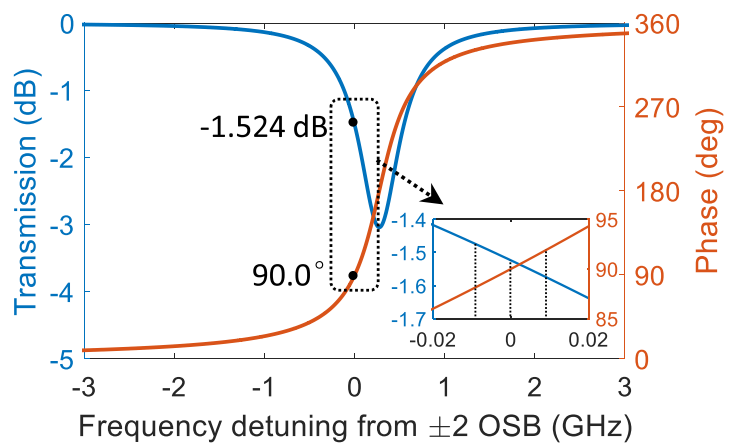

(b)

Fig.9. (a) Response of the ring resonators used to process the OCB. (b) Response of the ring resonators used to process the \pm 2 OSB. The insets are the zoom in figures at center of OCB and \pm 2 OSB [39].

meeting the condition in (9), causing incomplete cancellation of the IMD3 terms. The response of ring resonators used to process the optical spectrum are simulated in Fig.9. As depicted in Fig. 9, the phase shift and amplitude suppression are not constant in the processing bandwidth, leading to a degradation in IMD3 suppression.

In the experiment of serial linearization scheme, the UC ring (ring 4) that was used to process the optical carrier will heat up because of high optical power coupled into the ring. This temperature shift will lead to a resonance frequency shift due to both the thermo-optic effect and thermal expansion of the ring resonator. The frequency shift leads to reduction of the IMD3 suppression and also influences the stability in our system. In our setup, we use a thermoelectric cooler (TEC) controller to stabilize the temperature and reduce resonance frequency shift of the ring. The TEC module might overcompensate the thermal effect due to the lagging feedback from temperature change and create an oscillation in temperature. However the stability can be improved with a feedback controller that can tune the heaters on the ring resonators to compensate the frequency shift in realtime. As for the parallel linearization scheme, the optical carrier processing is implemented by de-interleaver and attenuator, which shows much improved stability in the experiment.

In parallel linearization methods, the imperfect IMD3 cancellation is mainly due to the nonlinear phase response in the passband of the de-interleaver. When we take nonlinear phase response into account, the photocurrent of IMD3 at output of BPD can be written as 


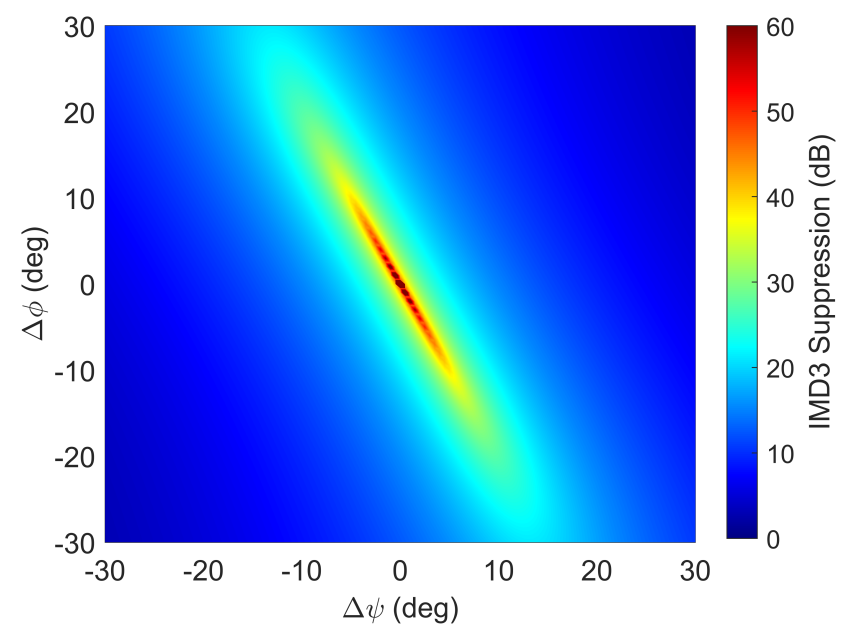

Fig. 10. Contour plot of the IMD3 suppression as a function of $\Delta \phi$ and $\Delta \psi$.

$$
I_{3}=\frac{m^{3}}{8} R_{p d} P_{i} T_{\text {pass }}^{2}\left[\begin{array}{l}
-\beta \cos \left(\left(2 \omega_{1,2}-\omega_{2,1}\right) t+\Delta \varphi_{\text {cross_1 }}\right) \\
-2 \beta \cos \left(\left(2 \omega_{1,2}-\omega_{2,1}\right) t+\Delta \varphi_{\text {cross_2 }}\right) \\
+\beta \cos \left(\left(2 \omega_{1,2}-\omega_{2,1}\right) t+\Delta \varphi_{\text {cross_3 } 3}\right) \\
+\cos \left(\left(2 \omega_{1,2}-\omega_{2,1}\right) t+\Delta \varphi_{\text {bar_1 }}\right)
\end{array}\right]
$$

In this case, $\Delta \varphi_{\text {cross } \_1}, \Delta \varphi_{\text {cross } \_2}, \Delta \varphi_{\text {cross } 3 \text {, }}$, and $\Delta \varphi_{\text {bar_1 }}$ are not identical, so even the condition of $\beta=1 / 2$ is met, the photocurrent of IMD3 is still not zero. As $\Delta \varphi_{\text {cross_1 }}$ and $\Delta \varphi_{\text {cross_2 }}$ both describe the phase difference between optical signals in OCB and +1 OSB imposed by de-interleaver, they can be regard as the same for simplicity. Then we simulate the influence of difference among $\Delta \varphi_{\text {cross_1 }_{1}} \Delta \varphi_{\text {cross_3 }}, \Delta \varphi_{\text {bar_1 }}$.

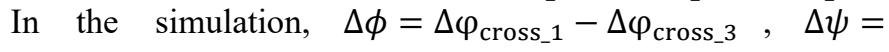
$\Delta \varphi_{\text {cross_1 }}-\Delta \varphi_{\text {bar_1 }}$.

Fig. 10 shows the contour plot of the IMD3 suppression as a function of $\Delta \phi$ and $\Delta \psi$. When $\Delta \phi=\Delta \psi=0$, which means the de-interleaver has a linear phase response in passband, IMD3 can be reduced more than $60 \mathrm{~dB}$. With the increase of $\Delta \phi$ and $\Delta \psi$, the IMD3 suppression decreased quickly. This shows that a more nonlinear phase response in pass-band will lead to a more degraded IMD3 suppression.

To further analyze the influence of the nonlinear phase response in the pass-band of de-interleaver, we fitted the measured de-interleaver response with the simulation model in (10), and used the de-interleaver model with fitted parameters to simulate the IMD3 suppression in the proposed parallel linearization methods. The fitting results and simulated phase response of de-interleaver are depicted in Fig.11(a) and (b). A nonlinear phase response can be observed at the edge of the passband. Fig. 11(c) is the simulated RF spectrum without linearization. Fig, 11 (d) is the RF spectrum after linearization using the de-interleaver with a nonlinear phase response. The IMD3 suppression is degraded to $8.6 \mathrm{~dB}$ compared to the ideal case of more than $60 \mathrm{~dB}$ suppression in Fig 10. This proves the influence of nonlinear phase response on IMD3 suppression.
To improve the IMD3 suppression, we can design a linear phase de-interleaver with multi-stage optical all-pass filters as phase compensator[43] or add a part of delay line in one path of parallel structure to add an additional phase shift to the IMD3 terms in one path to maintain the phase relation of destructive interference.

In the experiment, an IMD3 suppression of $20.3 \mathrm{~dB}$ is observed, which is better than the simulation results. This is because the fibers between the chip and BPD introduce a path length difference between two parallel paths, which works as a delay line and compensate the nonlinear phase response of the de-interleaver. The path length difference measured in the experimental setup is $1.6 \mathrm{~cm}$. Then a delay line is added to the lower sideband path in simulation model. With a $1.58 \mathrm{~cm}$ delay line, an IMD3 suppression of $20.1 \mathrm{~dB}$ is achieved, which fits with the experimental results. This illustrates that a proper length of delay line can compensate the nonlinear phase of deinterleaver and improve the IMD3 suppression.

Finally, a de-interleaver with linear phase response is used in the simulation model with equal path length. The magnitude response is the same as the response in Fig. 11(a), and the idea linear phase response is show in Fig. 11(b). As shown in Fig. 11(f), a linear phase de-interleaver will lead to a IMD3 suppression of more than $27.4 \mathrm{~dB}$. The isolation between two complementary channels of de-interleaver limits the further suppression of the IMD3.

As discussed above, the nonlinear phase response of deinterleaver and path length difference have a joint effect to the IMD3 suppression. To overcome this, we should design deinterleaver with more linear phase response and higher rejection, and integrate the BPD on chip to match the path length properly.

\section{B. Noise analysis}

The SFDR is influenced by both IMD3 suppression and the noise floor, namely the noise power spectral density (PSD) of the system. The noise sources in a MWP system mainly consist of thermal noise, shot noise, relative intensity noise (RIN), and amplified spontaneous emission from the EDFA [44].

There are fundamental differences in noise performance between serial and parallel linearization methods. First difference is related to the compensation of power penalty in fundamental signal. In serial spectral shaping linearization method, to meet the condition in (9), the phase shift at optical carrier is $340.5^{\circ}$ instead of $90^{\circ}$, leading to an incomplete PMIM conversion. So, there is a power penalty in fundamental signal although the optical power sent to PD is remained the same before and after linearization. To compensate this power penalty, the optical power sent to PD need to be increased, which elevates the noise floor from $-163.8 \mathrm{dBm} / \mathrm{Hz}$ to -155.1 $\mathrm{dBm} / \mathrm{Hz}$. However, in parallel spectral shaping method, the power penalty comes from the attenuation at upper sideband and optical carrier, which means the optical power send to PD is reduced after linearization. To compensate the reduced fundamental signal, the output power of EDFA is increased to keep the optical power at PD the same as the power without linearization. As the power sent to PD remains the same, there is not an increase in noise floor. 


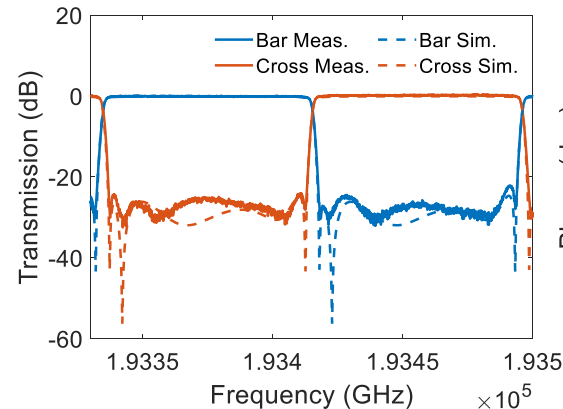

(a)

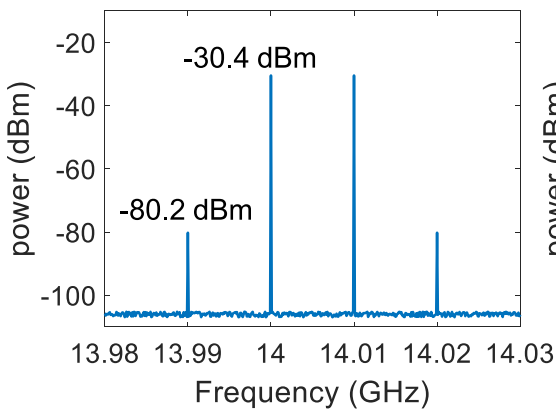

(d)

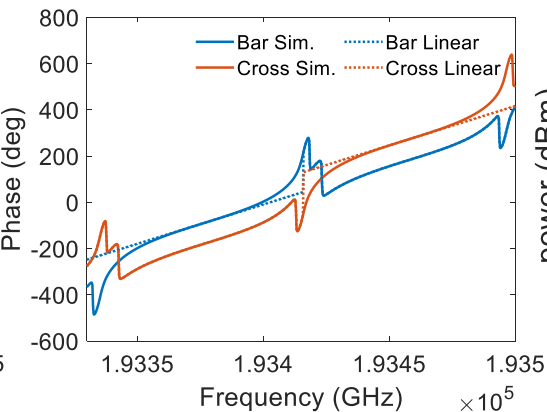

(b)

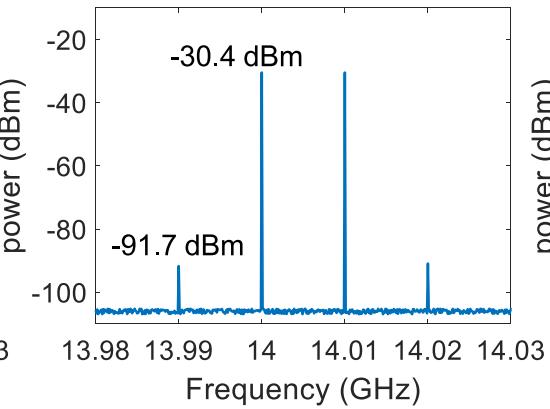

(e)

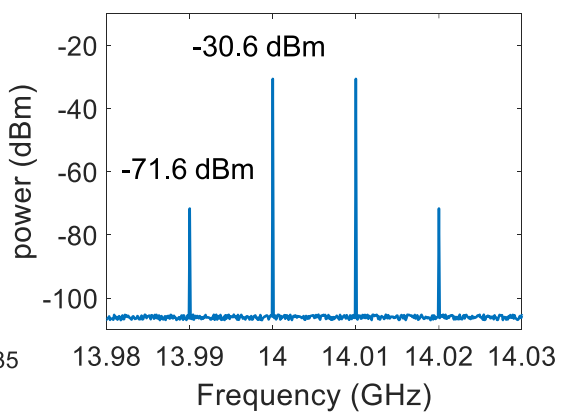

(c)

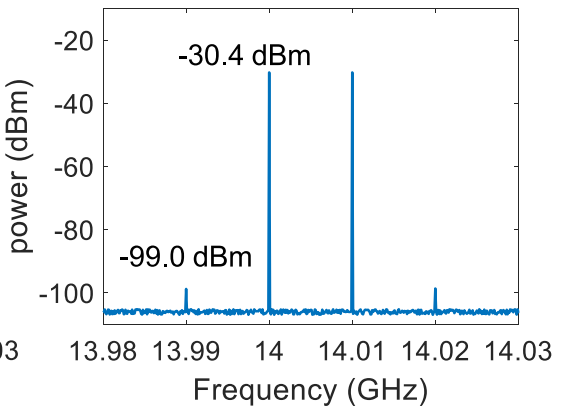

(f)

Fig. 11. (a) The measured and simulated de-interleaver magnitude response. (b) simulated phase response and idea linear phase response of de-interleaver (c)simulated RF spectrum before linearization (d) simulated RF spectrum after linearization (e) simulated RF spectrum with a path length difference of 1.58 $\mathrm{cm}$ between two parallel paths. (f) simulated RF spectrum with linear phase response in passband and equal path length.

The other difference is related to the principle of PM-IM conversion in two methods. In serial linearization method, an OC ring is used to process the optical carrier for PM-IM conversion. As the optical carrier is aligned at the slope of magnitude response of the ring, the ring resonator will convert phase noise of the laser to intensity noise, which adds an additional noise source to the system. However, in parallel linearization method, the PM-IM conversion is implemented by using de-interleaver to filter out the optical carrier and upper sideband. The optical carrier is completely in a passband of the de-interleaver, which has a flat response in magnitude, so there will not be any phase noise to intensity noise conversion. Based on the analysis above, in general, the spatial separated linearization scheme shows a better noise performance.

\section{CONCLUSION}

Two on-chip linearization methods based on integrated MWP spectral shaping technique are proposed and demonstrated. These methods show good IMD3 suppression of $22.4 \mathrm{~dB}$ and $20.3 \mathrm{~dB}$ respectively leading to an improved SFDR of $106.9 \mathrm{~dB} \cdot \mathrm{Hz}^{2 / 3}$ and $107.8 \mathrm{~dB} \cdot \mathrm{Hz}^{2 / 3}$. Currently, the performance is limited by the accuracy of phase and amplitude tailoring. Deep research still need to be done to find new structures and circuits for spectral shaping. The methods demonstrated in this paper, open the avenue to integrate the linearization and advanced functionalities on the same PIC, which will lead to ultra linear, low-loss and low noise advanced MWP systems.

\section{ACKNOWLEDGMENT}

The authors would like to thank Power Electronics \& EMC (PE) group in University of Twente for their kind help in measurement.

\section{REFERENCES}

[1] J. Capmany, and D. Novak, "Microwave photonics combines two worlds," Nature Photon., vol. 1, no. 6, pp. 319-330, Apr. 2007.

[2] J. Yao, "Microwave Photonics," J. Lightw. Technol., vol. 27, no. 3, pp. 314-335, Feb. 2009.

[3] J. Capmany, J. Mora, I. Gasulla, J. Sancho, J. Lloret, and S. Sales, "Microwave Photonic Signal Processing," J. Lightw. Technol., vol. 31, no. 4, pp. 571-586, Feb. 2013.

[4] D. Marpaung, J. Yao, and J. Capmany, "Integrated microwave photonics," Nature Photon., vol. 13, no. 2, pp. 80-90, 2019.

[5] D. Marpaung, C. Roeloffzen, R. Heideman, A. Leinse, S. Sales, and J. Capmany, "Integrated microwave photonics," Laser and Photon. Rev., vol. 7, no. 4, pp. 506-538, 2013.

[6] D. Marpaung, B. Morrison, R. Pant, C. Roeloffzen, A. Leinse, M. Hoekman, R. Heideman, and B. J. Eggleton, "Si3N4 ring resonator-based microwave photonic notch filter with an ultrahigh peak rejection," Opt. Exp., vol. 21, no. 20, pp. 23286-23294, Oct. 2013.

[7] Y. Liu, J. Hotten, A. Choudhary, B. J. Eggleton, and D. Marpaung, "Alloptimized integrated RF photonic notch filter," Opt Lett, vol. 42, no. 22, pp. 4631-4634, Nov. 2017.

[8] J. S. Fandiño, P. Muñoz, D. Doménech, and J. Capmany, "A monolithic integrated photonic microwave filter," Nature Photon., vol. 11, no. 2, pp. 124-129, 2016.

[9] L. Zhuang, C. G. H. Roeloffzen, M. Hoekman, K.-J. Boller, and A. J. Lowery, "Programmable photonic signal processor chip for radiofrequency applications," Optica, vol. 2, no. 10, pp. 854-859, Oct. 2015.

[10] D. Marpaung, B. Morrison, M. Pagani, R. Pant, D.-Y. Choi, B. LutherDavies, S. J. Madden, and B. J. Eggleton, "Low-power, chip-based stimulated Brillouin scattering microwave photonic filter with ultrahigh selectivity," Optica, vol. 2, no. 2, pp. 76-83, Feb. 2015. 
[11] Z. Zhu, Y. Liu, M. Merklein, O. Daulay, D. Marpaung, and B. J. Eggleton, "Positive link gain microwave photonic bandpass filter using Si3N4-ringenabled sideband filtering and carrier suppression," Opt. Exp., vol. 27, no. 22, pp. 31727-31740, Oct. 2019.

[12] O. Daulay, R. Botter, and D. Marpaung, "On-chip programmable microwave photonic filter with an integrated optical carrier processor," OSA Continuum, vol. 3, no. 8, pp. 2166-2174, Aug. 2020.

[13] O. Daulay, G. Liu, and D. Marpaung, "Microwave photonic notch filter with integrated phase-to-intensity modulation transformation and optical carrier suppression," Opt. Lett., vol. 46, no. 3, pp. 488-491, Feb. 2021.

[14] L. Zhuang, D. Marpaung, M. Burla, W. Beeker, A. Leinse, and C. Roeloffzen, "Low-loss, high-index-contrast $\mathrm{Si3N} 4 / \mathrm{SiO} 2$ optical waveguides for optical delay lines in microwave photonics signal processing," Opt. Exp., vol. 19, no. 23, pp. 23162-23170, Nov. 2011.

[15] Y. Liu, A. Choudhary, D. Marpaung, and B. J. Eggleton, "Gigahertz optical tuning of an on-chip radio frequency photonic delay line," Optica, vol. 4, no. 4, pp. 418-423, Apr. 2017.

[16] L. McKay, M. Merklein, A. C. Bedoya, A. Choudhary, M. Jenkins, C. Middleton, A. Cramer, J. Devenport, A. Klee, R. DeSalvo, and B. J. Eggleton, "Brillouin-based phase shifter in a silicon waveguide," Optica, vol. 6, no. 7, pp. 907-913, Jul. 2019.

[17] C. Zhu, L. Lu, W. Shan, W. Xu, G. Zhou, L. Zhou, and J. Chen, "Silicon integrated microwave photonic beamformer," Optica, vol. 7, no. 9, pp. 1162-1170, Sep. 2020.

[18] M. H. Khan, H. Shen, Y. Xuan, L. Zhao, S. Xiao, D. E. Leaird, A. M. Weiner, and M. Qi, "Ultrabroad-bandwidth arbitrary radiofrequency waveform generation with a silicon photonic chip-based spectral shaper," Nature Photon., vol. 4, no. 2, Art. no. 2, Feb. 2010.

[19] D. Marpaung, L. Chevalier, M. Burla, and C. Roeloffzen, "Impulse radio ultrawideband pulse shaper based on a programmable photonic chip frequency discriminator," Opt. Exp., vol. 19, no. 25, p. 24838, Dec. 2011.

[20] J. Wang, H. Shen, L. Fan, R. Wu, B. Niu, L. T. Varghese, Y. Xuan, D. E. Leaird, X. Wang, F. Gan, A. M. Weiner, and M. Qi, "Reconfigurable radio-frequency arbitrary waveforms synthesized in a silicon photonic chip," Nature Commun., vol. 6, no. 1, Art. no. 1, Jan. 2015.

[21] Z. Zhu, Y. Liu, M. Merklein, Z. Zhang, D. Marpaung, and B. J. Eggleton, "Si3N4-chip-based versatile photonic RF waveform generator with a wide tuning range of repetition rate," Opt. Lett., vol. 45, no. 6, pp. 13701373, Mar. 2020.

[22] H. Jiang, D. Marpaung, M. Pagani, K. Vu, D.-Y. Choi, S. J. Madden, L. Yan, and B. J. Eggleton, "Wide-range, high-precision multiple microwave frequency measurement using a chip-based photonic Brillouin filter," Optica, vol. 3, no. 1, pp. 30-34, Jan. 2016.

[23] M. Burla, X. Wang, M. Li, L. Chrostowski, and J. Azaña, "Wideband dynamic microwave frequency identification system using a low-power ultracompact silicon photonic chip," Nature Commun., vol. 7, no. 1, Art. no. 1, Sep. 2016.

[24] D. Marpaung, C. Roeloffzen, A. Leinse, and M. Hoekman, "A photonic chip based frequency discriminator for a high performance microwave photonic link," Opt. Exp., vol. 18, no. 26, pp. 27359-27370, Dec. 2010.

[25] E. I. Ackerman, "Broad-band linearization of a Mach-Zehnder electrooptic modulator," IEEE Trans. Microw. Theory and Tech., vol. 47, no. 12 , pp. 2271-2279, Dec. 1999.

[26] B. M. Haas, V. J. Urick, J. D. McKinney, and T. E. Murphy, "DualWavelength Linearization of Optically Phase-Modulated Analog Microwave Signals," J. Lightw. Technol., vol. 26, no. 15, pp. 2748-2753, Aug. 2008.

[27] W. Zhang, A. Wen, X. Xu, W. Zhai, K. Wei, and H. Zhang, "Dualwavelength linearization of analog photonic link based on PM-IM conversion," Opt. Commun., vol. 420, pp. 174-178, Aug. 2018.

[28] J. Dai, K. Xu, R. Duan, Y. Cui, J. Wu, and J. Lin, "Optical linearization for intensity-modulated analog links employing equivalent incoherent combination technique," in Proc. Int. Topical Meet. Microw. Photon. Conf., Oct. 2011, pp. 230-233.

[29] M. Huang, J. Fu, and S. Pan, "Linearized analog photonic links based on a dual-parallel polarization modulator," Opt. Lett., vol. 37, no. 11, p. 1823, Jun. 2012.

[30] Y. Cui, Y. Dai, F. Yin, J. Dai, K. Xu, J. Li, and J. Lin, "Intermodulation distortion suppression for intensity-modulated analog fiber-optic link incorporating optical carrier band processing," Opt. Exp., vol. 21, no. 20, p. 23433 , Oct. 2013.

[31] P. Li, L. Yan, T. Zhou, W. Li, Z. Chen, W. Pan, and B. Luo, "Improvement of linearity in phase-modulated analog photonic link," Opt. Lett., vol. 38, no. 14, p. 2391, Jul. 2013.
[32] J. Li, Y. Zhang, S. Yu, and W. Gu, "Optical Sideband Processing Approach for Highly Linear Phase-Modulation/Direct-Detection Microwave Photonics Link," IEEE Photon. J, vol. 6, no. 5, pp. 1-10, 2014.

[33] W. Zhu, J. Hu, Y. Gu, F. Fan, Z. Kang, X. Han, and M. Zhao, "Dynamic Range Improvement of a Microwave Photonic Link Based on Brillouin Processing," IEEE Photon. Technol. Lett., vol. 28, no. 23, pp. 2681-2684, Dec. 2016.

[34] R. Wu, T. Jiang, S. Yu, J. Shang, and W. Gu, "Multi-Order Nonlinear Distortions Analysis and Suppression in Phase Modulation Microwave Photonics Link," J. Lightw. Technol., vol. 37, no. 24, pp. 5973-5981, Dec. 2019

[35] Y. Gu, and J. Yao, "Microwave Photonic Link With Improved Dynamic Range Through $\pi$ Phase Shift of the Optical Carrier Band," J. Lightw. Technol., vol. 37, no. 3, pp. 964-970, 2019.

[36] J. Zhang, A. Wang, L. Yu, P. Du, W. Cong, J. Zhang, and J. Wo, "Dynamic range improvement for a microwave photonic link using a phase modulator," in 17th Int. Conf. on Opt. Commun. and Net. (ICOCN2018), 2019, vol. 11048, pp. 59-62.

[37] O. Daulay, G. Liu, X. Guo, M. Eijkel, and D. Marpaung, "A Tutorial on Integrated Microwave Photonic Spectral Shaping," J. Lightw. Technol., pp. $1-1,2020$

[38] X. Guo, Y. Liu, T. Yin, B. Morrison, M. Pagani, O. Daulay, W. Bogaerts, B. J. Eggleton, A. Casas-Bedoya, and D. Marpaung, "Versatile silicon microwave photonic spectral shaper," APL Photon., vol. 6, no. 3, p. 036106, Mar. 2021.

[39] G. Liu, O. Daulay, Q. Tan, H. Yu, and D. Marpaung, "Linearized phase modulated microwave photonic link based on integrated ring resonators," Opt. Exp., vol. 28, no. 26, pp. 38603-38615, Dec. 2020.

[40] C. G. H. Roeloffzen, M. Hoekman, E. J. Klein, L. S. Wevers, R. B. Timens, D. Marchenko, D. Geskus, R. Dekker, A. Alippi, R. Grootjans, A. van Rees, R. M. Oldenbeuving, J. P. Epping, R. G. Heideman, K. Wörhoff, A. Leinse, D. Geuzebroek, E. Schreuder, P. W. L. van Dijk, I. Visscher, C. Taddei, Y. Fan, C. Taballione, Y. Liu, D. Marpaung, L. Zhuang, M. Benelajla, and K. Boller, "Low-Loss Si3N4 TriPleX Optical Waveguides: Technology and Applications Overview," IEEE J. Sel. Top. in Quantum Electron., vol. 24, no. 4, pp. 1-21, 2018.

[41] D.A.I. Marpaung, "High dynamic range analog photonic links: Design and implementation," Ph.D. dissertation, Dept. Telecommun. Group, Faculty Elect. Eng., Math., Comput. Sci., Univ. of Twente, Enschede, The Netherlands, 2009.

[42] L.-W. Luo, S. Ibrahim, A. Nitkowski, Z. Ding, C. B. Poitras, S. J. B. Yoo, and M. Lipson, "High bandwidth on-chip silicon photonic interleaver," Opt. Exp., vol. 18, no. 22, pp. 23079-23087, Oct. 2010.

[43] C. K. Madsen, and J. H. Zhao, Optical Filter Design and Analysis: A Signal Processing Approach. John Wiley \& Sons, 1999.

[44] V. J. Urick Jr, J. D. Mckinney, and K. J. Williams, Fundamentals of Microwave Photonics. USA: John Wiley \& Sons, 2015.

Gaojian Liu received his B.Eng. degree in electronic information engineering from Zhejiang University, Hangzhou, China in 2014, and the M.S. degree in electronic science and technology from China Academy of Space Technology (CAST), Xi'an, China, in 2017. He is currently pursuing a Ph.D. degree at CAST. Now He is working as a visiting Ph.D. student with the Nonlinear Nanophotonics Group - LPNO, University of Twente, the Netherlands. His current research interests include microwave photonic signal processing and linearization.

Okky Daulay received his B.Eng. degree in electrical engineering from National Defense Academy, Yokosuka, Japan in 2009, and the M.S. degree in embedded systems from University of Twente, Enschede, the Netherlands, in 2018. He is currently working towards a Ph.D. degree at the Nonlinear Nanophotonics Group - LPNO, University of Twente. His research interests include microwave engineering and RF 
photonics in all their aspects and phased-array technology. He is an officer and a researcher at Department of Research and Development of Indonesian Air Force. He is responsible for research and development of Radar, Avionics, Communication and Electronic Warfare technology.

Yvan Klaver received his M.Sc. degree in applied physics from the University of Twente, Enschede, The Netherlands, in 2020. Currently he is pursuing a Ph.D. degree at the Nonlinear Nanophotonics - Laser Physics and Nonlinear Optics group, University of Twente. His current research interests include integrated optical nonlinear resonators.

Roel Botter received his M.Sc. degree in applied physics from the Delft University of Technology, the Netherlands, in 2018. $\mathrm{He}$ is currently pursuing a Ph.D. degree at the Nonlinear Nanophotonics - Laser Physics and Nonlinear Optics group, University of Twente. His current research interests include stimulated Brillouin scattering and other acousto-optic interactions in integrated waveguides.

Qinggui Tan is a research professor with National Key Laboratory of Science and Technology on Space Microwave, China Academy of Space Technology (Xi'an), Xi'an, China. His current research interests include microwave phonics systems and optical satellite communications.

Hongxi Yu is a research professor with China Academy of Space Technology (Xi'an), Xi'an, China. His current research interests include microwave and millimeter wave circuits and systems, microwave phonics systems and satellite communications.

Marcel Hoekman studied applied physics at the University of Twente, Enschede, The Netherlands, and received the Graduate degree from the Integrated Optical Micro Systems Group, University of Twente, in 1998. From 2004 to 2009, he has been a part-time Ph.D. student with the Integrated Optical Micro Systems Group, University of Twente in the framework of the STW project "Multi-Sensing Arrays of Separately Accessible Optics Sensors." He finished a Post Graduate Design Program at the Integrated Optical Micro Systems Group that was based on the design of an integrated optical electro-optical modulator based on PZT containing multilayer-stacks, in 2001. In this project, he worked together with the group Experimental Solid State Physics III, Radboud University Nijmegen, The Netherlands. He joined Lion Photonix Technologies BV, in 2001, which was renamed to LioniX BV since 2002 and to LioniX International BV since 2016-as a Design Engineer. Besides simulation and mask design, he has experience in several cleanroom manufacturing processes and characterization methods, and he has worked as a Project Leader on several projects.

Edwin J. Klein received the M.Sc. degree from the Department of Electrical Engineering, University of Twente, Enschede, The Netherlands, in 2002 and received the Ph.D. degree from the Integrated Optical MicroSystems Group, University of Twente, in 2008. He was closely involved in the founding of LioniX
International Photonics B.V., where he was working as the Vice-President of Research \& Development since 2008. He has (co)authored more than 45 publications in journals and conferences and (co)holds five patents. His research interests include the development and practical application of complex photonic devices at visible and near-infrared wavelengths.

David Marpaung received his Ph.D. degree in electrical engineering from the University of Twente, The Netherlands, in 2009. From 2009 to 2012 he was a postdoctoral researcher in the University of Twente, working on microwave photonic system integration for optical beamforming. He joined CUDOS University of Sydney, Australia in August 2012 as a research fellow. From 2015 to 2017 he was a senior research fellow leading the nonlinear integrated microwave photonics research activities in CUDOS University of Sydney.

In 2018, he joined the University of Twente as an associate professor leading the Nonlinear Nanophotonics Group - LPNO, where he was promoted to Professor in 2020. Prof. Marpaung was the recipient of the 2015 Discovery Early Career Research Award (DECRA) from the Australian Research Council, the 2017 Vidi award, and the 2018 Start Up fellowship from the Netherlands Organization for Scientific Research (NWO). His research interests include RF photonics, optomechanics, nonlinear optics, and phononics. 